\title{
Experimental Thermodynamics of the Helix-Random Coil Transition. II. Calorimetric Measurement of Transition Enthalpies of Poly A in Acid Aqueous Solution
}

H. KLUMP, TH. ACKERMANN, and E. NEUMANN, Institut für Physikalische Chemie der Universität Münster (Westfalen), Germany

\section{Synopsis}

The enthalpy change accompanying the double helix-coil transition of polyriboadenylic acid (poly $\mathrm{A}$ ) in aqueous solution has been measured optically and calorimetrically in the $\mathrm{pH}$ range 5.7-4.5. The course of this cooperative transition was followed optically by measuring changes in ultraviolet absorption as a function of temperature at different $\mathrm{pH}$ values, and calorimetrically by determining the heat capacity of the solution through the transition region. From the latter measurements, the enthalpy of transition was calculated. It is shown, that $\Delta H$ is dependent on $\mathrm{pH}$ as it is expected from the influence of protonation of the double helix of poly $\mathrm{A}$.

\section{INTRODUCTION}

Hydrodynamic and other physico-chemical studies of solutions of polyriboadenylic acid (poly A) have shown, that this polynucleotide can undergo reversibly a type of helix-coil transition. ${ }^{1}$ The randomly coiled* form is stable at neutral $\mathrm{pH}$, where it predominantly exists as a single-stranded, flexible, rodlike conformation that is stabilized by stacking of the adenine bases. $^{2-7}$ On lowering the $\mathrm{pH}$ so that at least $65 \%$ of the adenine groups have been titrated, the net negative charge is reduced sufficiently to permit the single chain to associate to form organized linear complexes of varying length. These are negatively birefringent, highly extended, slightly flexible particles with hydrodynamic properties similar to those of undenatured deoxyribonucleic acid (DNA). ${ }^{8}$ The molecular properties of this form have been interpreted in terms of a double-stranded helical model with discontinuities where one strand ends and another begins. Rich and coworkers $^{9}$ found $\mathrm{x}$-ray evidence, that the acid-stable aggregates had an ordered helical structure and postulated, that this could be identified with the double-stranded helical structure proposed by Fresco. ${ }^{10}$ Recently the optical rotatory dispersion (ORD), the absorbance, and the viscosity of

* We will later use the term randomly coiled interchangeably with one-stranded, although the degree of randomness of the single strand remains to be established. 
poly A have been measured by Holcomb and Tinoco ${ }^{3}$ as a function of temperature and $\mathrm{pH}$. Analysis of the data has led to the suggestion, that the $\mathrm{pH} 7$ conformation is a flexible single-stranded helix with stacked bases and that the low $\mathrm{pH}$ double-strand helix dissociates to this single-strand helix at high temperature. The viscosity studies of poly $\mathbf{A}$ are in general agreement with the optical studies except that they are complicated by aggregation phenomena. Thus the rise in viscosity at $\mathrm{pH} 5.4$ before the sharp drop which agrees with the optical transition temperature is probably due to the dissociation of large aggregates of the double-strand helices. A similar effect occurs at $\mathrm{pH} 5.0$, while at $\mathrm{pH} 4.6$ the aggregation is so pronounced, that the specific viscosity is nearly zero. Unfortunately, the alteration in physical properties accompanying the protonation of adenine presents a far from clearcut picture. Some increase in molecular weight is always observed. Its magnitude increases sharply with increasing concentration of poly A." Under the appropriate conditions, increases in molecular weight of tenfold or more are observed." These special difficulties have to be watched carefully when calorimetric experiments are carried out in acid $\mathrm{pH}$ region.

As in all calculations of $\Delta H$ values from data obtained in optical measurements it is necessary to make some sort of extrapolation to determine the value of the measured quantities for the complete helix and the complete random coil, it is therefore useful to determine the apparent heat of transition experimentally by calorimetric measurements.

\section{EXPERIMENTAL}

\section{Materials}

The potassium salt of poly A used in this investigation was purchased from Miles Chemical Company (lot number 110638) and was used without further purification. All other materials were commercial preparations of analytical reagent grade. Poly A solutions were prepared by first dissolving the required amounts of poly $\mathrm{A}$ and $\mathrm{NaCl}$ (all buffers and solutions contain $0.15 M \mathrm{NaCl}$ ) in distilled water and then adding $0.2 M$ sodium citrate buffer to yield the desired $\mathrm{pH} .{ }^{12}$ Variations of $\mathrm{pH}$ were obtained by dropwise addition of $\mathrm{HCl}$.

\section{Measurements}

Ultraviolet Absorption Measurements. Ultraviolet absorption measurements were performed with a Zeiss PMQII spectrophotometer. Absorption data of the poly A solution were obtained at various temperatures with the use of buffer of the same concentration of cations, $\mathrm{pH}$, and temperature as the blank. The temperature of the cell holder was kept constant to 0.1 degree using a Colora thermostat. All $\mathrm{pH}$ measurements were made at room temperature with a Radiometer Type $22 \mathrm{pH}$ meter. 
Calorimetric Measurements. A new adiabatic semimicro calorimeter with a working capacity of $80-100 \mathrm{ml}$ has been developed especially for thermodynamic investigations of biological polymers. This calorimeter is an improved version of the original model, which we have used in a series of earlier investigations. ${ }^{13-15}$ The calorimeter vessel was filled with ca. $80 \mathrm{ml}$ of a dilute aqueous solution of poly $\mathrm{A}$ at citrate-buffered acid $\mathrm{pH}$ and continuously heated by applying constant electric power (permanently controlled). The heat capacity data were calculated from the measured $\Delta T$ values according to the eq. (1)

$$
C_{(\mathbf{T})}=U^{2} \Delta t / R \Delta T
$$

(where $C_{(\mathrm{T})}$ is the heat capacity, $U$ is the voltage drop across the electric heater, $R$ is the resistance of the heater, and $\Delta T$ is the temperature difference corresponding to the time interval $\Delta t$ ) and recorded as a function of temperature (see Fig. 3). Since the amount of the additional heat capacity to be measured during the thermal change of the secondary structure is very small, possibly less than $1 \%$ of the total heat capacity of the polymer solution, it is necessary to measure the heat capacity over an appropriate temperature range with extreme accuracy. As $U^{2} / R$ is the constant electric power continuously fed into the heating elements of the calorimeter vessel the determination of $C_{(\mathrm{T})}$ is reduced to the measurement of the temperature change $\Delta T$ for a given time interval $\Delta t$. In this experiment, temperature was measured by means of a Hewlett-Packard quartz thermometer ${ }_{16,17}$ to $0.0001^{\circ} \mathrm{C}$. The maximum fluctuation of the difference of two subsequent temperatures registered was $\pm 1 \times 10^{-4}$ degree and is mainly caused by fluctuation of the residual heat flow between the vessel and the adiabatic shield. Thus it is possible to obtain the heat capacity data of a solution of polymers to an accuracy of about $0.001 \mathrm{cal} / \mathrm{ml}-\mathrm{deg}$ and the additional heat capacity data caused by the helix-coil transition of the dissolved polynucleotide to about $5 \%$ over a temperature range of about $70^{\circ} \mathrm{C}$. The additional enthalpy change $\Delta H$, accompanying this thermal conversion, thus can be determined within the margin of experimental error of at most $5 \%$.

\section{RESULTS}

Figure 1 shows the ultraviolet absorption of poly $A$ as a function of temperature at different $\mathrm{pH}$ values at $2520 \AA$. The usual increase of nearultraviolet absorption due to a structural change of the polynucleotide was observed. The fact that all curves join the same ascending line suggests that a portion of stacking interactions is preserved under the double helixcoil transition and that the remaining portion is almost noncooperatively unstacked with increasing temperature. However, it can also be attributed to the temperature-dependent hypochromicity of nonbonded nucleotides. ${ }^{18}$ The dependence of the transition temperature $T_{m}$ on $\mathrm{pH}$ is shown in Figure 2. Open circles indicate data from absorption measure- 


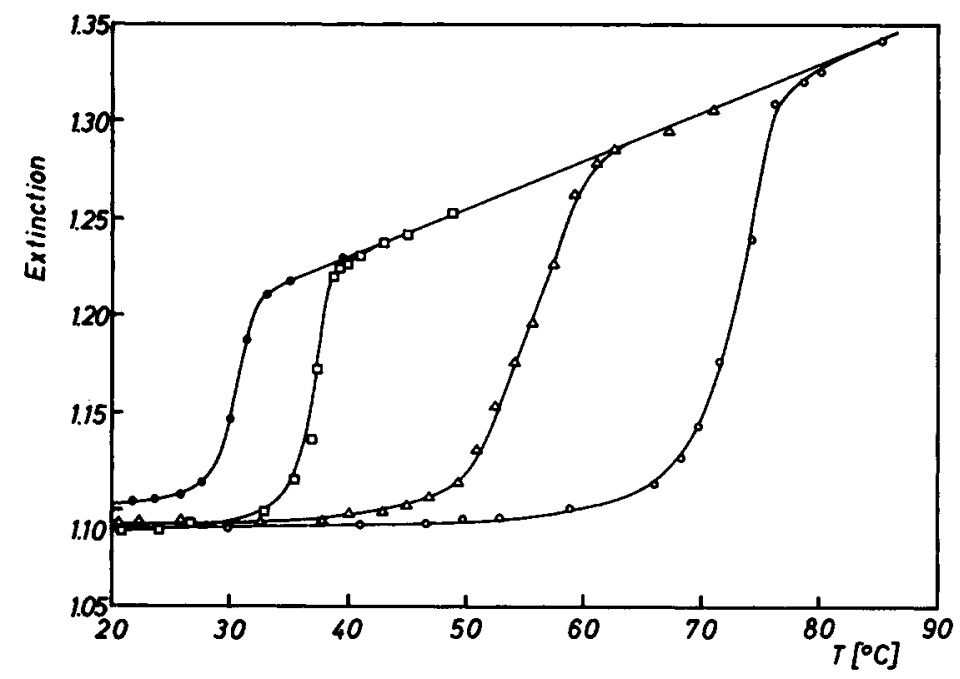

Fig. 1. Ultraviolet absorption as a function of temperature at various $\mathrm{pH}$ values at

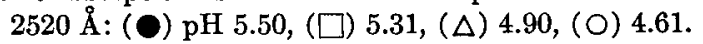

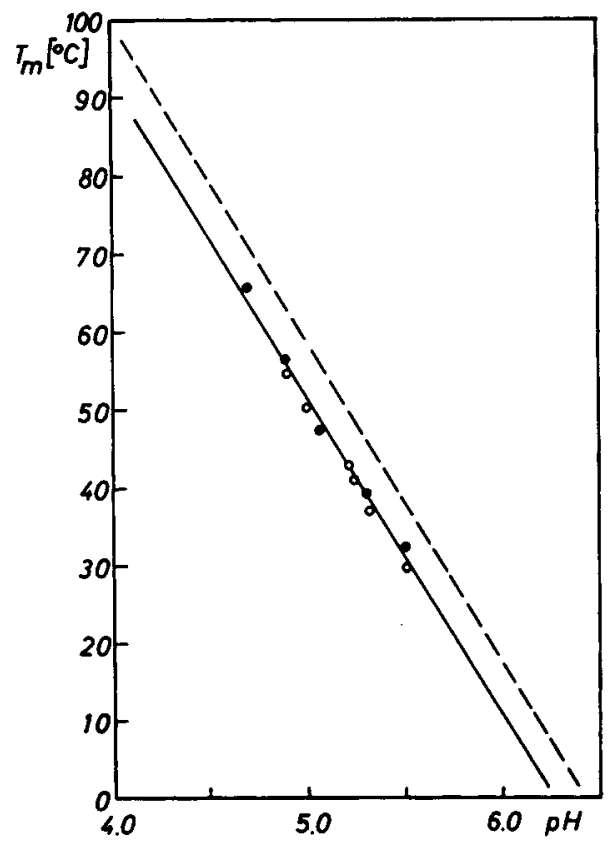

Fig. 2. Transition temperature $T_{m}$ as a function of $\mathrm{pH}$ : (O) data obtained calorimetrically; $(O)$ data obtained by ultraviolet absorption measurements. A similar plot has been presented by Holcomb and Tinoco ${ }^{3}(--\infty)$ and Massoulie. ${ }^{20}$ 


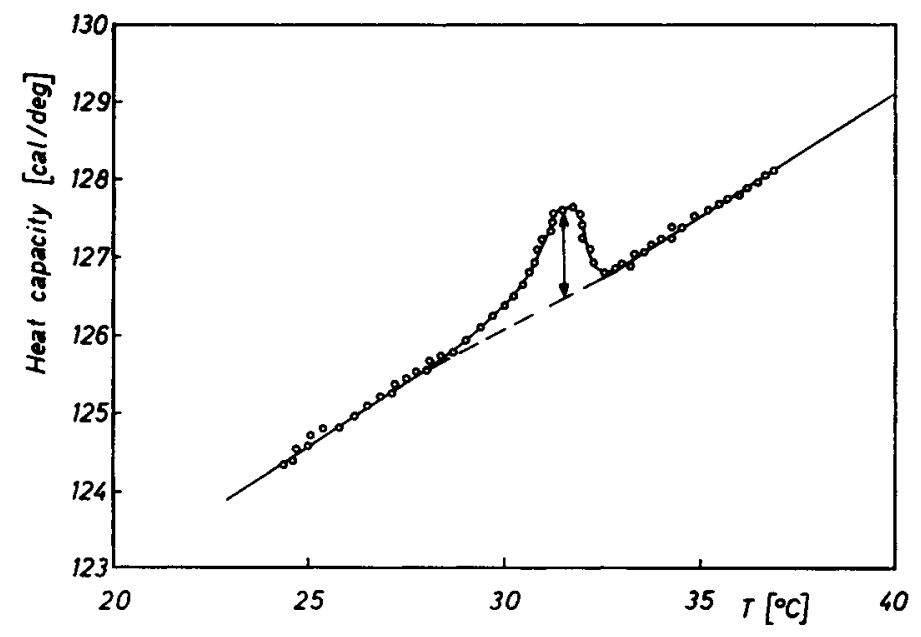

Fig. 3. Heat capacity of an acid aqueous poly $A$ solution $(0.15 M \mathrm{NaCl}, 0.2 M$ citrate buffer, $\mathrm{pH}$ 5.5) as a function of temperature. Polymer concentration $c=6.76 \times 10^{-3}$ mole base pair $/ 1000 \mathrm{~g}$ solution.

ments and full circles show those from calorimetric measurements. A similar plot has previously been presented by Holcomb and Tinoco ${ }^{3}$ and by 'Ts'o. ${ }^{19}$ They found a linear relationship of the same slope but displaced to the right (to higher $\mathrm{pH}$ ) by $0.2 \mathrm{pH}$ units. As Massoulie ${ }^{20}$ shows. this is due to lower salt concentration used by these authors, as $T_{m}$ shows a dependence upon salt concentration while $\mathrm{pH}$ is kept constant. The double-strand helix is stable to the left of the straight line while the random coil is stable to the right. Figure 3 shows the heat capacity of the dilute poly A solution measured over a $15^{\circ} \mathrm{C}$ range centered about $T_{m}$, a heating rate of about $3^{\circ} \mathrm{C} / \mathrm{hr}$ being used. A maximum in the heat capacity at $T_{m}$ was observed. This is in excellent agreement with the half-conversion temperature $T_{m}$ determined by ultraviolet absorption. The excess heat capacity $\Delta C_{p}$ referred to a smoothed baseline can be obtained as a function of temperature from Figure 3 . The area under this curve is proportional to the apparent transition enthalpy $\Delta H$ of the polynucleotide. $\Delta H$ can be calculated from the calorimetric data according to the equation

$$
\Delta H=\int_{0}^{\infty} \Delta C_{p} d T
$$

With increasing $\mathrm{pH}$ the $\Delta H$ values increase. In Figure 4 the $\Delta H$ values are plotted against $T_{m}$ and are extrapolated to a half conversion temperature of $90-100^{\circ} \mathrm{C}$. At this temperature the bases of poly $A$ are fully unstacked and there is no short- or long-range order left in the macromolecule. ${ }^{21}$ The $\Delta H$ value obtained in this way amounts to $5.6 \mathrm{kcal} / \mathrm{mole}$ base pair. The accuracy of the $\Delta C_{p}$ values is proved by two different methods. First 


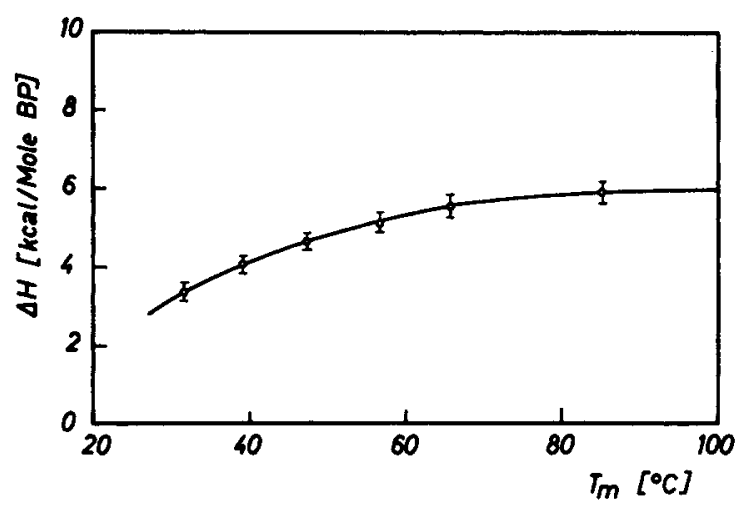

Fig. 4. Transition enthalpy $\Delta H$ as a function of temperature $T_{m}$, extrapolated to $T_{m}=100^{\circ} \mathrm{C}$.

the heat capacity of a buffer solution was measured and showed a straight line as expected. When the weight of the solution was altered by adding $2.0 \mathrm{~g}$ water, the heat capacity increases by exactly $2 \mathrm{cal} /{ }^{\circ} \mathrm{C}$. The second method was to alter the heating energy stepwise as if an enthalpy change were occurring. The $\Delta C_{p}$ values obtained by employing this arrangement were plotted against temperature. The alteration of $\Delta C_{p}$ shows a characteristic maximum. The area under this curve is proportional to $\Delta H$ and can be referred exactly to the variation of the heating power. From these calibration tests it is reasonable to assume that the results obtained by the calorimetric experiments are exact within the margin of experimental error as stated earlier in this paper.

\section{DISCUSSION}

Our results show an increase of the $\Delta H$ values from $3.36 \pm 0.30 \mathrm{kcal} / \mathrm{mole}$ base pair at $\mathrm{pH} 5.50$ to $5.57 \pm 0.50 \mathrm{kcal} / \mathrm{mole}$ at $\mathrm{pH} \mathrm{4.70}$. The total enthalpy change is correlated with the following processes, all having a share in the double helix-coil transition: breaking of hydrogen bonds; separation of the strands, each assuming a randon coil configuration, in which the individual purine and pyrimidine residues are individually immersed in the aqueous solvent and hydrogen bond with the solvent; abolishing of stacking interactions as well as deprotonation and changes in the intensity of electrostatic forces. Most of the enthalpy change is associated with the stacking interaction. The total energy of the hydrogen bonding interaction is close to zero, because hydrogen bonds of the base pairs ${ }^{22}$ must be broken in order to form the base-water hydrogen bonds.*

* Up to the present time none of the experimental techniques applied in this field is capable of revealing detailed information on the various contributions to the stabilization energies resulting from different processes. NMR measurements as performed by $\mathrm{McD}$ onald and Phillips ${ }^{21}$ may help to resolve this problem. 
For this reason, the stacking interactions are assumed to give the major contribution to the helix stabilization energies. They result both from the electronic interaction energy between the two stacked base pairs and also from the tendency of the solvent (water) to force the bases together.

This latter is the so-called hydrophobic interaction. The contribution of the solvent to the free energy of stacking is not yet fully understood. The stability of the coil form at high temperature results from its high entropy relative to the helix. Freedom of rotation around many bonds is gained in the disorganization of the helix and at sufficient high temperature the resulting entropy compensates the unfavorable energy in the transition. ${ }^{23}$ Changes in the degree of ionization or protonation could not be measured separately, but it is assumed that their amount gives only a minor contribution to the transition enthalpy. The increase of transition temperature $T_{m}$ with increasing acidity can be taken as a hint that the electrostatic repulsion of the negatively charged phosphate groups of the backbone of the polymer is diminsished by the added electrolyte and/or protons, so that the strands are able to combine more perfectly. Hereby the possibility for base stacking interactions is improved. An increase of the $\Delta H$ values would then result from an increase of stacking interaction. This is in good agreement with our experimental results (Table I).

TABLE I

Calorimetric $\Delta \boldsymbol{H}$ Values Accompanying the Conformational Change of Poly A in Acid Aqueous Solution ${ }^{\mathrm{a}}$

\begin{tabular}{ccc}
$\mathrm{pH}$ & $\begin{array}{c}T_{m}, \\
{ }^{\circ} \mathrm{C}\end{array}$ & $\begin{array}{c}\Delta H, \\
\mathrm{kcal} / \mathrm{mole} \text { base pair }\end{array}$ \\
\hline 5.50 & 31.5 & 3.36 \\
5.30 & 39.2 & 4.09 \\
5.06 & 47.1 & 4.67 \\
4.89 & 56.6 & 5.13 \\
4.70 & 65.5 & 5.57 \\
4.20 & 85.5 & $5.90^{\mathrm{b}}$ \\
\hline
\end{tabular}

${ }^{s} c=0.00676$ mole base pair $/ 1000 \mathrm{~g}$ solvent; solvent: $0.20 \mathrm{M}$ citrate; $0.15 \mathrm{M} \mathrm{NaCl}$ and HCl.

b The last value was obtained later in a measurement performed to justify the extrapolation to $T_{m} 90-100^{\circ} \mathrm{C}$.

Some remarks should be included here regarding the results of various authors ${ }^{18,24,25}$ who have also performed calorimetric investigations of the double helix-coil transition of poly $\mathbf{A}$ in acid aqueous solution. However, these measurements must be regarded to correspond to different processes occurring in this system, and a direct comparison of the results will easily lead to some confusion. The data of Rawitscher et al. ${ }^{24}$ correspond to the heat envolved when $\mathrm{HCl}$ is added to a solution of poly $\mathrm{A}$ initially at $\mathrm{pH} 7$, and the authors thereby obtained the enthalpy of the reaction

$$
\mathrm{N}(\text { coil })+\alpha \mathrm{H}^{+}=\mathrm{NH}_{\alpha}(\text { helix })
$$


where $\mathbf{N}$ represents a nonprotonated nucleotide residue and $\alpha$ is the fraction of adenine groups protonated at $\mathrm{pH} 4.00$ in the helical form. The numerical value of this enthalpy change is $-3.4 \mathrm{kcal}$. Fresco et al. ${ }^{25}$ have studied a somewhat different process occurring in the buffered system, namely

$$
\mathrm{NH}_{\alpha^{\prime}}(\text { coil })+\left(\alpha-\alpha^{\prime}\right) \mathrm{HCt}=\mathrm{NH}_{\alpha}(\text { helix })+\left(\alpha-\alpha^{\prime}\right) \mathrm{Ct}^{-}
$$

where $\alpha^{\prime}$ is the fraction of adenine groups protonated at $\mathrm{pH} 4.00$ in the randomly coiled form, and HCt represents citric acid. The enthalpy change for this reaction amounts to $-4.0 \pm 0.4 \mathrm{kcal}$.

The $\Delta H$ values computed by Applequist and Damle from Fresco's hypochromicity data are -7.98 for the staggering zipper model, -7.48 for the nonstaggering zipper model, and -6.59 for the all-or-none model. ${ }^{18}$ Since these results are computed from the slopes of the relative hypochromicity versus temperature curves, which were not measured at the characteristic wavelength of the absroption maximum for the poly A helix ( $2520 \AA$ ) but at $2570 \AA$, they may not be regarded as quantitative with respect to the margin of experimental error.

It is doubtful that a meaningful comparison could be made from the data mentioned above, and we will not attempt it here, since the enthalpies of ionization of adenine in both the helix and the random coil and of citric acid are not known. Our own value of $5.57 \pm 0.50 \mathrm{kcal} / \mathrm{mole}$ base pairs does not measure the enthalphy change corresponding to the bonding forces in the helix solely but measures correctly the total energetic contribution to the stability of the helix under the conditions of the experiment. Since Massoulie ${ }^{20}$ and $\mathrm{Ts}^{\prime} \mathrm{o}^{19}$ have shown in recent papers that the salt concentration plays an important role in systems of the type discussed here, we intend further heat capacity measurements on solutions of poly $\mathrm{A}$ at various salt concentrations.

\section{References}

1. J. R. Fresco and P. Doty, J. Amer. Chem. Soc., 79, 3928 (1957).

2. V. Luzzati, A. Mathis, F. Masson, and J. Witz, J. Mol. Biol., 10, 28 (1964).

3. D. N. Holcomb and I. Tinoco, Jr., Biopolymers, 3, 121 (1965).

4. K. E. Van Holde, J. Brahms, and A. Michelson, J. Mol. Biol., 12, 726 (1965).

5. A. Michelson, T. L. V. Ulbricht, T. R. Emerson, and R. J. Swan, Nature, 209, 873 (1966).

6. J. Brahms, A. Michelson, and K. E. Van Holde, J. Mol. Biol., 15, 467 (1966).

7. M. Leng and G. Felsenfeld, J. Mol. Eiol., 15, 455 (1966).

8. P. Doty, B. B. MeGill, and S. Rice, Proc. Nat. Acad. Sci. U.S., 44, 432 (1958).

9. J. R. Fresco, J. Mol. Biol., 1, 106 (1959).

10. A. Rich, D. R. Davies, F. H. C. Crick, and J. D. Watson, J. Mol. Biol., 3, 71 (1961).

11. R. F. Steiner and R. F. Beers, Polynucleotides, Elsevier, Amsterdam, 1961, Chap. 8, Tab. 26.

12. J. R. Fresco and E. Klemperer, Ann. N. Y. Acad. Sci., 81, 730 (1959).

13. Th. Ackermann, Z. Elektrochem., 62, 411 (1958).

14. A. Eucken and M. Eigen, Z. Elektrochem., 55, 343 (1951).

15. F. Schreiner, Thesis, Hamburg, 1959. 
16. A. Benjaminson (Hewlett-Packard), The Quartz Crystal Resonator as a Linear Digital Thermometer. 4th Annual Conference of the Temperature Measurements Society, Feb. 1965.

17. D. L. Hammond, C. A. Adams and P. Schmidt, paper presented at 19th Annual ISA conference, New York, Oct. 1964; Reprint No. 11, 2-3-64.

18. J. Applequist and V. Damle, J. Amer. Chem. Soc., 87, 1450 (1965).

19. P. O. P. Ts'o, G. K. Helmcamp, and C. Sanders, Proc. Nat. Acad. Sci. U.S., 48, 686 (1962).

20. M. J. Massoulie, Compt. Rend., 260, 5554 (1965).

21. C. C. McDonald and W. D. Phillips, Magnetic Resonance in Biological Systems, Pergamon Press, London, p. 4.

22. N. Davidson, in The Neurosciences, G. C. Quarton, Ed., The Rockefeller University Press, New Yo k, 1968.

23. D. M. Crothers, in The Neurosciences G. C. Quarton, Ed. The Rockefeller University Press, New York, 1968.

24. M. A. Rawitscher, P. D. Ross, and J. M. Sturtevant, J. Amer. Chem. Soc., 85, 1915 (1963).

25. J. R. Fresco, R. Blake, and P. Doty, in preparation.

Received June 24, 1968

Revised October 1, 1968 